University of Wollongong

Research Online

Faculty of Informatics - Papers (Archive)

Faculty of Engineering and Information

Sciences

2010

\title{
Multimedia adaptation based on semantics from social network users interacting with media
}

\author{
S. J. Davis \\ University of Wollongong, stdavis@uow.edu.au \\ Eva Cheng \\ RMIT University \\ I. Burnett \\ Faculty of Informatics, University of Wollongong, ianb@uow.edu.au \\ Christian Ritz \\ University of Wollongong, critz@uow.edu.au
}

Follow this and additional works at: https://ro.uow.edu.au/infopapers

Part of the Physical Sciences and Mathematics Commons

\section{Recommended Citation}

Davis, S. J.; Cheng, Eva; Burnett, I.; and Ritz, Christian: Multimedia adaptation based on semantics from social network users interacting with media 2010.

https://ro.uow.edu.au/infopapers/3425

Research Online is the open access institutional repository for the University of Wollongong. For further information contact the UOW Library: research-pubs@uow.edu.au 


\title{
Multimedia adaptation based on semantics from social network users interacting with media
}

\begin{abstract}
A key goal of adaptive multimedia delivery is to provide users with content that maximizes their quality of experience. To achieve this goal, adaptive multimedia systems require descriptions of the content and user preference information, moving beyond traditional criteria such as quality of service requirements or perceptual quality based on traditional metrics. Media is increasingly consumed within online social networks and multimedia sharing websites can also add a wealth of metadata. In this paper, mechanisms for gathering semantics that relate to user preferences when interacting with media content in social networks are proposed. Subjective results indicate the proposed mechanisms can successfully provide information about user and social group media preferences that can be used for adapting multimedia for improved user quality of experience.

\section{Disciplines}

Physical Sciences and Mathematics

\section{Publication Details}

S. J. Davis, E. C. Cheng, I. S. Burnett \& C. H. Ritz, "Multimedia adaptation based on semantics from social network users interacting with media," in 2010 Second International Workshop on Quality of Multimedia Experience (QoMEX): Proceedings, 2010, pp. 170-175.
\end{abstract}




\title{
MULTIMEDIA ADAPTATION BASED ON SEMANTICS FROM SOCIAL NETWORK USERS INTERACTING WITH MEDIA
}

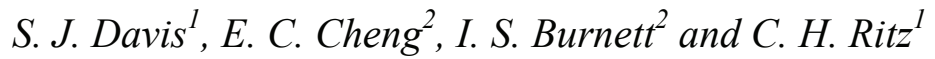 \\ ${ }^{1}$ School of Electrical, Computer and Telecommunications Engineering, University of Wollongong \\ ${ }^{2}$ School of Electrical and Computer Engineering, RMIT University
}

\begin{abstract}
A key goal of adaptive multimedia delivery is to provide users with content that maximizes their quality of experience. To achieve this goal, adaptive multimedia systems require descriptions of the content and user preference information, moving beyond traditional criteria such as quality of service requirements or perceptual quality based on traditional metrics. Media is increasingly consumed within online social networks and multimedia sharing websites can also add a wealth of metadata. In this paper, mechanisms for gathering semantics that relate to user preferences when interacting with media content in social networks are proposed. Subjective results indicate the proposed mechanisms can successfully provide information about user and social group media preferences that can be used for adapting multimedia for improved user quality of experience.
\end{abstract}

Index Terms - Multimedia Semantics, Adaptive Multimedia Delivery, Social Networking, Tagging, User Behavior

\section{INTRODUCTION}

The increasing popularity of multimedia sharing sites (such as YouTube [1]) has led to the availability of a large number of videos, both professional content and User Generated Content (UGC) via the web. Simultaneously, social networking sites (such as Facebook [2], MySpace [3]) have seen increases in user numbers, users sharing multimedia content with friends, and users accessing social media though mobile devices [4]. Thus, media adaptation has become commonplace as mobile network operators and/or multimedia sharing sites modify the content presentation to maximize user quality of experience (QoE) e.g., different display resolution, dropping packets, perceptual encoding, and bitrate adjustment - often in response to Quality of Service (QoS) metrics. QoE, however, is comprised of two main components [5]: Quality of Service (QoS) and Quality of Perception (QoP). Whilst QoS characterizes the technical perspectives that relate to multimedia distribution, QoP pertains to a user's perspective that requires user feedback or interaction to measure the content and quality [5]. A good QoE therefore requires a balance between QoS and QoP. QoS has been well studied [6][7][8] and there are existing QoE approaches and frameworks [9][10][11], however, these systems do not collect the user's QoP semantic metadata required to describe the user's perspective to feed back into the adaptation system. Such QoP semantic metadata can be used not only adapt multimedia for different users, but for a user's time-varying, content and device dependent quality preferences. Semantic metadata that relate to a user's multimedia QoE, however, are both highly subjective, unique to an individual [12]. Further, QoE metrics also depend on the user's motivation, intent and purpose for viewing the content. However, the increasing prevalence of users accessing multimedia through social networks [4] introduces the opportunity to improve a user's experience through multimedia adaptation based on semantics derived from the user's behavior and preferences as exhibited from media interactions within social networks.

Social networking sites have now become dominant on the Internet [4] and a user's consumption of media within social networks provides a unique vantage point when classifying users as many preferences are often shared between users within certain social groups. In addition, many users volunteer information about their preferences in their social networking user profile (e.g., favorite music, movies, books, etc). Thus, even without any form of 'training', an adaptive system can recommend a better experience based on access to a user's social groups and any volunteered information about preferences and interests. However, social media user profiles only provide a glimpse into what a user considers enjoyable, thus an end-user tool incorporated into social media that collects the semantics of a user's and their social groups' interaction with multimedia is also required.

This paper builds upon the authors' previous work [13], which utilized a Flex-based application 'Tag!t' (in this paper deployed as a Facebook application) to facilitate the collection of semantics relating to users' interactions with media. Specifically, this paper studies the use of semantics derived from user media preferences, where user preferences can be inferred from users' interactions with media e.g., tagging media content within social networks. Through an analysis of the user's tags and tagging behavior within social groups, the collected semantics can then be used to enhance the QoE of the user and the user's social groups when using multimedia and the 'wisdom of crowds' in social media.

\section{SEMANTICS FROM SOCIAL NETWORK USERS INTERACTING WITH MEDIA}

Fig. 1 illustrates the framework of a system that can derive semantics from a range of collected user experience-based QoP metadata. Such semantic metadata can then be used to drive a multimedia adaptation system to improve users' multimedia quality of experience (QoE). Fig. 1 loosely groups the different types of QoE metadata into four categories:

- User media preferences e.g., video interaction (start/stop/pause etc.) and tagging (like/dislike/funny etc.) 


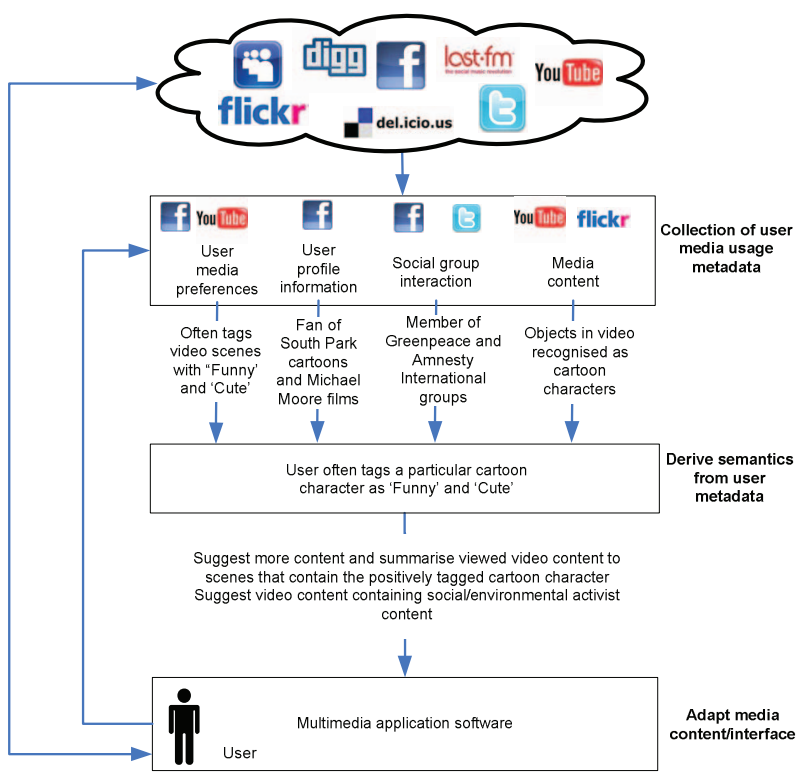

Fig. 1. Multimedia Adaptation using QoE Semantic Metadata

- User profile information e.g., from social network user profiles

- Social group interaction e.g., collaborative tagging activity

- Media content-based metadata e.g., signal processing analysis

\subsection{User Media Preferences}

Observing how a user interacts with the media can build semantics that relate to a user's media preferences e.g., a user may prefer to skip over certain parts of videos rather than watching clips in entirety, which many indicate their preference for those scenes in the video (especially when combined with the tagging of media). However, whilst media access metadata (i.e., video start/stop/pause) can indicate video scene preferences, video tagging is a popular activity from which it is possible to gain comprehensive insights into how a user feels about media: tags can include emotion tags (e.g., happy, sad) and tags in response to another person or viewpoint raised in the media (e.g., agree and disagree). The frequency of tagging within a clip also indicates information about a user's media preferences e.g., this might show specific parts of the video the user really enjoys. Further, studying a user's tagging vocabulary may also reveal the user's expertise and experience on various topics (e.g., a user tagging content with technical terms).

\subsection{User Profile Information}

A user's social networking profile can be used to derive vital information about a particular user and their interests e.g., favorite music, colors, movies, books, etc. To facilitate the collection of such user profile metadata from social media, many social media sites offer public APIs to interact with their services e.g., integrate social networking into applications and extract relationships about users and their friends/groups. For example, the Facebook API allows Facebook applications to access information about the user and depending on the user's privacy settings, an application can also gain access to a user's friends, their groups, posted links, notes and even their photos. With such knowledge about a user's interests and hobbies, an adaptive multimedia system can thus suggest topically relevant media to the user.

\subsection{Social Group Interaction}

In addition to allowing access to a user's profile, the social networking APIs provide information about the user's friends and group memberships. As users group together with their friends and others with similar interests in social networks (e.g., photography, sports etc.), such groups can be exploited to discover a user's interaction within social groups and group preferences related to media content consumption. Thus, to augment individual user preferences, the combination of a user's group memberships and analysis of group interactions with media content can reveal further social context-based media usage preferences.

\subsection{Media Content-Based Metadata}

To complement user social networking profile or social group interaction metadata, semantics related to a user's preferred type of media content are also required to comprehensively build a user's media interaction profile (see Fig. 1). Such media content-based metadata is often exposed through public APIs on multimedia sharing sites such as YouTube [1] and Flickr [14]. These APIs allow an application to search their extensive media collections and retrieve detailed information about photos and videos (e.g., resolution, video length, number of views), as well as the author tags and user ratings and comments. Further, signal processing techniques can also be leveraged to provide further metadata about the content e.g., analyzing the visuals, audio and subtitles.

\subsection{QoE Multimedia Adaption with Semantic Metadata}

Comprehensive QoE semantics can thus be derived from building on the different types of QoE metadata described in Sections 2.12.4. For the example user in Fig. 1, media content metadata can be extracted using object/face detection to recognize a particular cartoon character, but combining this content-based metadata with a user's tagging activity (i.e., user media preference metadata) can thus build semantic metadata: that the user finds this character particularly cute and funny. Thus, media content that contains this character can then be suggested to the user or a customized video summary can be generated on-the-fly composed only of the scenes featuring this particular character. Then, an analysis the information that the user has volunteered on their social network profile can further reveal that this user is a member of Amnesty International and Greenpeace groups and a fan of South Park cartoons and Michael Moore films. A QoE system enabled with knowledge of the user's interests can then suggest relevant media content to the user (to share with their groups), inferring that this user is likely to prefer social comments or environmental activist media content rather than content about the latest celebrity gossip. 


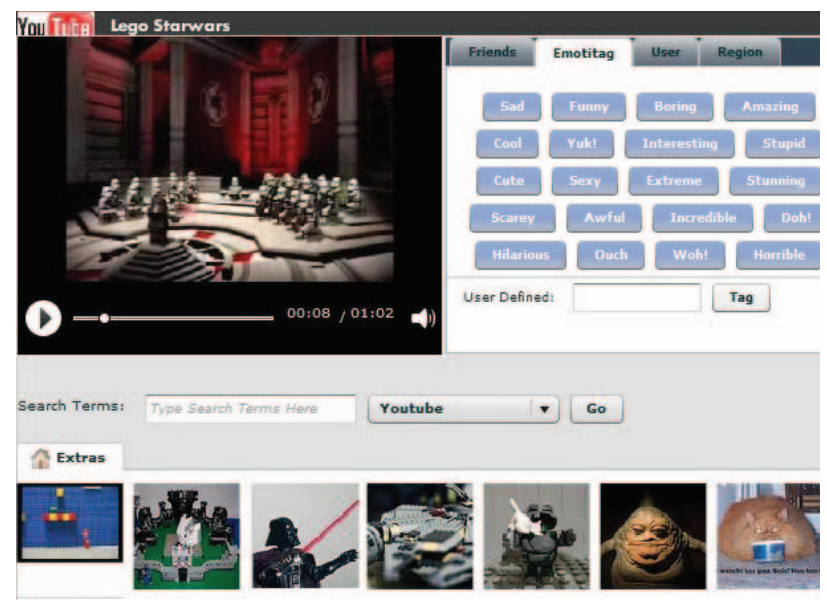

Fig. 2. Screenshot of the Tag!t client. The user is presented with a video panel, a tagging panel, an extras panel and a search box.

\section{A TOOL FOR GATHERING USER MEDIA PREFERENCE SEMANTICS}

This paper focuses on the collection and use of user media preference metadata (see Section 2.1) from users' interaction with media within social networks as the first step of a comprehensive QoPbased multimedia adaptation system for improved user QoE (see Fig. 1). The collection of such user media preference metadata within social networks requires a new technology tool, developed as 'Tag!t' by the authors [13]. While the authors' previous work investigated the collection of semantics from users' interactions with media using Tag!t [13], this paper specifically focuses on the use of the collected user preference metadata to build semantic metadata that can be utilized to adapt multimedia in QoE systems.

\subsection{How Tag!t Works}

Tag!t is a Flex-based Facebook application, developed to allow users to tag multimedia directly from their web browser. Tag!t exploits social media usage trends by tapping into the Facebook social network to gather information about a user's interests and preferences through their social network profile and social groups and interactions. To augment such user metadata collected from Facebook and also address the variability in semantics from multimedia tagging, Tag!t also leverages social networks to build upon the strength of many users contributing tags to a segment of media content: social networks bring social relationships and interactivity to collaborative tagging, which can lead to new tag characteristics and tagging behaviors [15]. This approach taken by Tag!t [13] thus provides a basis for the extraction of semantic metadata through observations of how different users and social groups interact with multimedia.

Fig. 2 shows a screenshot of the Tag!t client user interface, where the user is presented with a video panel, a tagging panel, an 'extras' panel (to display related content returned by the Tag!t system) and a search box (where users can type in terms to find extras). Tag!t extracts information both explicitly (i.e., through direct user input) and implicitly (i.e., automated without user in-

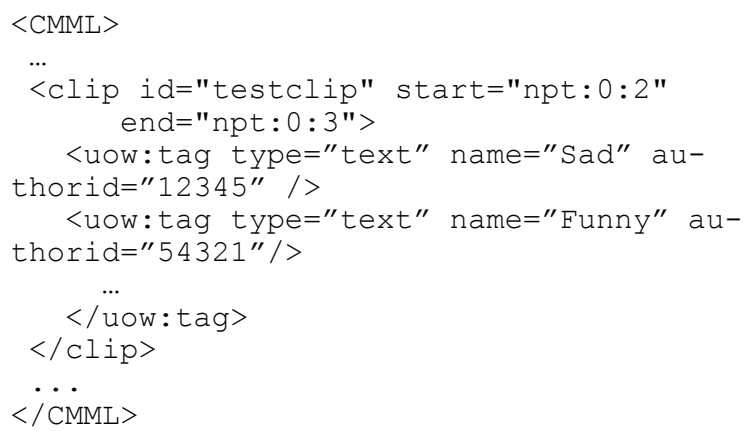

Fig. 3. Example CMML document illustrating how an tags are sent to the client.

put), which can be fed back into a QoE system. Semantics are derived from metadata collected using the following techniques [13]:

- Tagging - users can tag the video by simply clicking their chosen tag at any time while viewing the video;

- Search Events - whenever a user performs a search, the search terms and the timestamp of the video is recorded. This allows Tag!t to determine if anything in that scene is related to the search terms;

- Linked Content - after users have searched for and found relevant content, they may attach it to the current video since semantics derived from the linked content may be relevant to the current video; and

- Usage Behavior - user usage behaviors such as pausing and seeking within a video may provide valuable information about the content and user's interaction with the video.

The internal mechanisms of the Tag!t system are as follows: when a video is played in the client, the server retrieves all tags related to that video (and optionally can filter tags) and injects them into a Continuous Media Markup Language (CMML) [16] document. CMML provides a standardized way to time-synchronize markup with media, whereby the schema has been extended to support Tag!t (i.e., to support tags and tag sets). The client receives and parses the CMML document and display the tag to the user at the specified time. Fig. 3 shows an example CMML fragment defining 2 tags at timestamp 3. For each metadata type collected (tagging, search events, linked content and usage behavior), the video's current timestamp (in seconds) is also recorded and then sent to the server.

\subsection{Example Tag!t Usage for QoE Multimedia Adaptation}

While Tag!t was designed to allow the authors to collect semantic metadata from user interaction with video content, it has also opened some interesting application directions such as the use of such semantic metadata for multimedia adaptation in QoE systems. As this paper focuses on the collection and use of user preference metadata (Section 2.1), only the temporal content tagging capabilities of Tag!t are utilized. Further, as Tag!t is deployed as a Facebook application in this paper, different user tagging behaviors can be observed for individual users as well as within social group contexts. As an example Tag!t usage scenario, imagine that a user who is running the Tag!t Facebook application watches a YouTube 


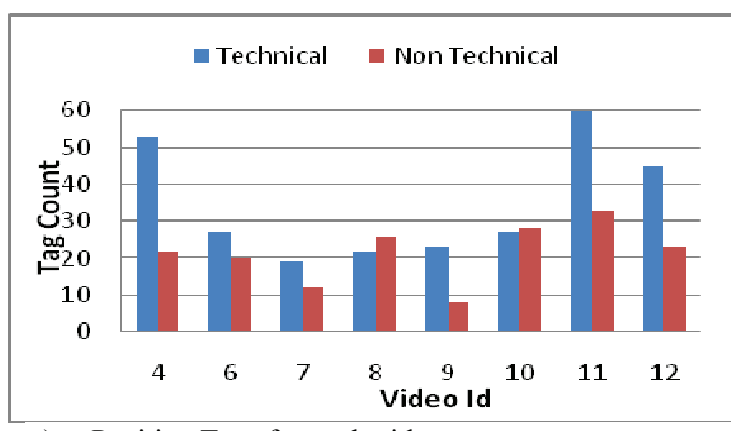

a) Positive Tags for each video

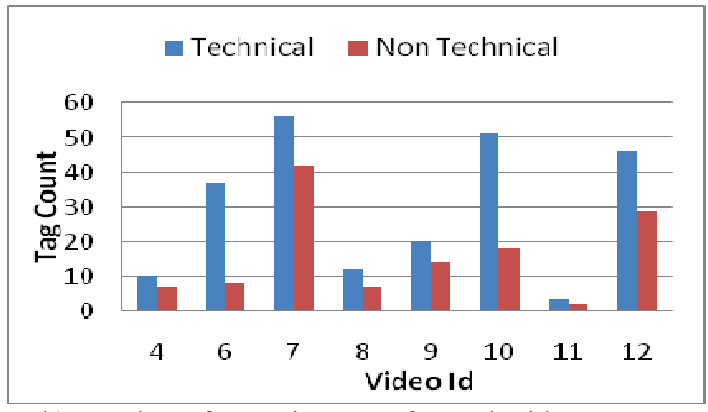

b) Number of Negative Tags for each video

Fig. 4. Comparison between the positive and negative tags for each video by technical and non-technical groups

video of an animation depicting Darth Vader conducting a band of Storm Troopers playing his theme from Star Wars using Tag!t (see Fig. 2). While watching the video, the user tags a few scenes as 'Funny' and 'Like'. Given the positive tags that the user has entered for the certain scenes in the video, Tag!t returns to the user an automatically generated summary of the video containing only the scenes the user tagged positively. When the user is finished viewing and tagging a few videos, Tag!t uses the Facebook news feed to alert the user's friends that the user has watched and tagged the clips. The story continues with the viral spread of the videos, user tags, and linked content generated using Tag!t. It is important to note the natural integration provided by the Tag!t application between social networking (via Facebook) and the user interface with the video content. The scenario purely describes the collection of semantic metadata, but it is a small step to change Tag!t for the subsequent users, based on the experience and behavior of the first user, since that is all captured within the CMML event data.

\section{EXPERIMENTAL OBSERVATIONS}

\subsection{User Experiments}

To evaluate user media preference metadata acquisition using the Tag!t system, an experiment was conducted where users were asked to tag eight short video clips ranging in duration from 68 seconds to 124 seconds. The videos included genres such as comedy, documentary, news, sports, science, and music (for further information about the experiment see [13] ). All participants held University degrees or were current University students and of the 22 people that participated in the experiment, 59\% classified themselves as technical and $41 \%$ as non-technical [13].

In the user experiments, Tag!t was deployed with a static tag set, with the choice of tags presented to the user limited to 20 'emotitags' that expressed emotions such as 'funny', 'cool', and 'boring'. For this experiment, users were not allowed to create new userdefined tags as the experiment aimed to observe the social groupings apparent with a limited (and manageable) set of tags. This paper focuses on analyzing the results from the experiment in [13] to investigate the use of user preference metadata (see Section 2.1) in the context of multimedia adaption for QoE.

\subsection{Results Evaluation}

From the 22 participants a total of 860 tags were collected across the eight videos in the experiment, and users were asked to identify with either the 'technical' or 'non-technical' social group. Most notably, there were distinct differences between the tagging behavior and response to the videos between the technical and nontechnical groups. In the following results, the video clips that were used can be summarized as follows:

- Vid 4 - A short clip showing some fancy street football moves;

- Vid 6 - An educational and comical rap about the Large Hadron Collider (LHC) at CERN;

- Vid 7 - A compilation of the top 5 laziest inventions;

- Vid 8 - A musical mash-up incorporating music videos created by other users on YouTube;

- Vid 9 - A compilation of the top 5 warring robots;

- Vid 10 - A short comedy scene from a American sitcom about physicists;

- Vid 11 - A compilation of scenes from the New Scientist; and

- Vid 12 - A compilation of the top 5 animal scenes in sports

To reduce variability in the tag semantics for results analysis, the 20 emotitags presented to participants in Tag!t were classed into 10 positive and 10 negative tags: positive tags included emotitags such as 'funny', 'cool', 'cute', and 'sexy', whilst negative tags included 'sad', 'boring', 'horrible', 'scary', and 'stupid'.

\subsection{Tagging Behaviour Results}

Fig. 4 illustrates the number of positive and negative tags that users tagged for each video, where vid 7 clearly stands out with the most negative tags while vids 4 and 11 accumulated the most positive tags. To study the effect of the occupation-based social groups (technical and non-technical), Fig. 4 shows the number of positive and negative tags for each video according to the two groups. The results in Fig. 4 suggest differences in tagging behavior between the two social groups: despite vids 6 and 10 being quite technical in content, the technical group negatively tagged both videos suggesting that they did not enjoy the technical comedies presented. The non-technical group, however, tagged vids 6 and 10 more positively rather than negatively. In contrast, both the technical and non-technical groups disliked vid 7 and enjoyed vid 11, where technical participants were more likely to tag the New Scientist video (vid 11) and the non-technical users did not tag as much but still liked it. The different tagging behaviors exhibited between the two social groups indicate that different social groups do interact 

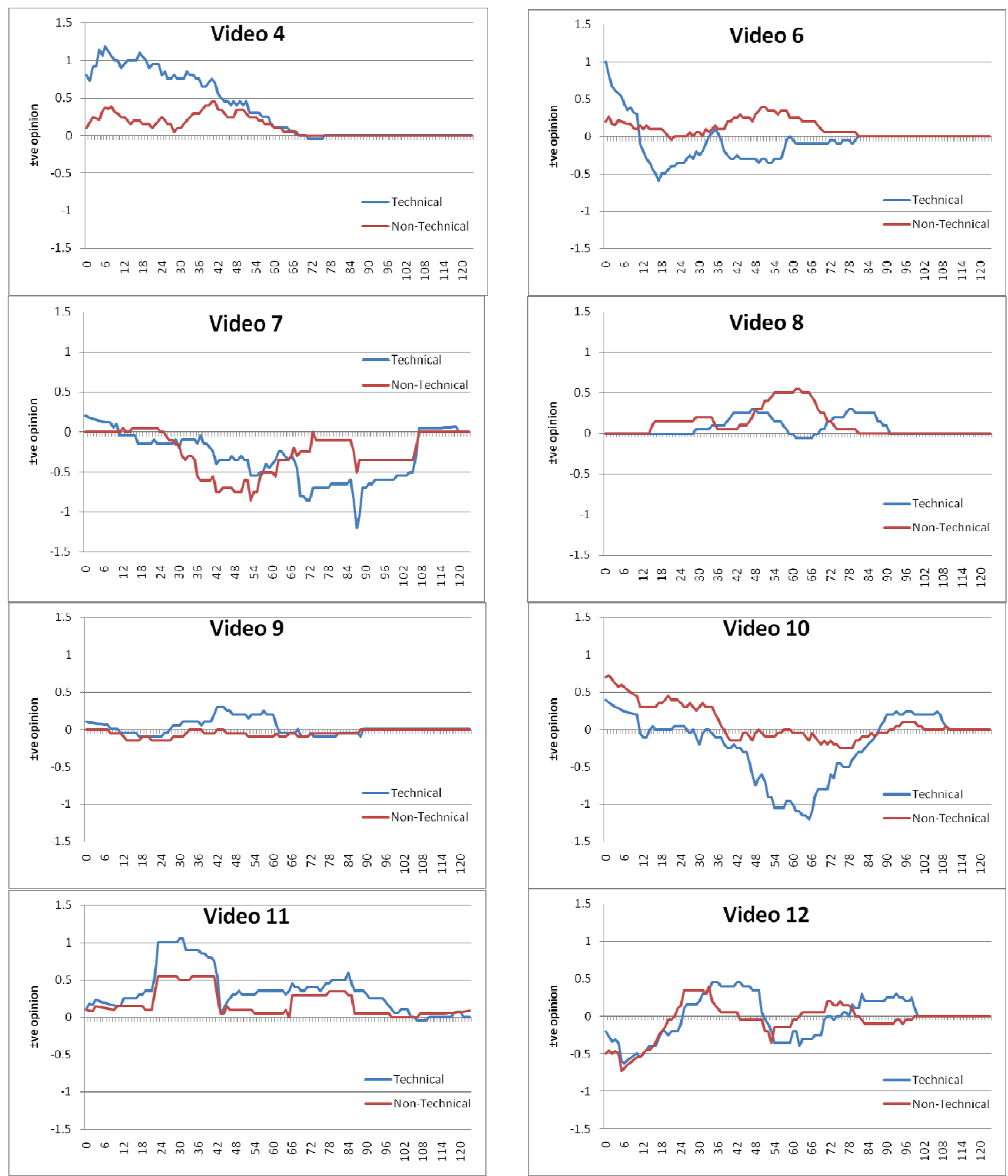

Fig. 5. Plots of positive and negative temporal trends for each video illustrating user tagging nature

and react to the same media differently; thus, metadata collected from user interactions in social contexts using Tag!t can be used for QoE multimedia adaptation. Further, differences in the groups' tagging behaviors are independent of content related to the group's common interest/s: even when presented with general interest media content (i.e., videos such as $4,7,8$ and 12 , where content is unspecific to the technical/non-technical nature of either group), the tagging behaviors between the technical and non-technical groups are still dissimilar.

Overall, from Fig. 4 it can be seen that the non-technical users tended to add fewer tags: on average, there were 33 tags per nontechnical user as opposed to 42 tags per technical user [13]. This discrepancy could be due to technical users being more familiar with the task of tagging media content, where non-technical users could be persuaded to tag more with a simpler user interface or simpler tag set - adaptive multimedia interfaces could thus be utilized to improve the QoE and better engage users of less technical capability.

\subsection{Temporal Tagging Results}

To represent the user 'opinion' throughout a video, we define a measure for average user opinion at time $l, O_{l}$ as follows: 


$$
O_{l}=\frac{1}{N} \sum_{n=l-N / 2}^{l+N / 2} w(n)\left(T_{p}(n)-T_{n}(n)\right)
$$

where $T_{p}(n)$ and $T_{p}(n)$ represent the total number of positive and negative tags, respectively, contributed by all users at time $n$ in the video, and $N$ is the segment length over which the average is calculated (here $N=20$ ). The weights, $w(n)$, define a sliding window equal to the segment length. Here, we use a square window (where $w(n)=1$ within the segment and 0 outside) and $l$ and $n$ have a resolution of 1 second.

Fig. 5 shows the results from applying (1) to the tags for each video according to the two social groups of technical/non-technical users. Results show that there are videos with peaks corresponding to both negative and positive opinions, highlighting the fact that temporal tagging is a useful metric in monitoring how a user's feeling changes during the video. For example, Fig. 5 shows similar opinion trends for both social groups for vid 10, where segments in the first 30 seconds that were tagged positively, while user opinion becomes negative between 40-85 seconds before again ramping up to positive from 85 seconds onwards. Vid 11 in Fig. 5 also shows temporal variation in user response: there are three spikes in the video that correspond to each of the three separate segments within the clip, where people enjoyed the last and the first segments more than the second segment.

Such temporally variable user responses to media content suggests that simply tagging and categorizing whole videos (as in Fig. 4) may not be a fine enough temporal resolution for effective QoE multimedia adaptation. Hence, by allowing users to temporally tag within videos, a QoE adaptive multimedia system can then gauge which parts of the video a user (or group of users) particularly enjoy. Thus, video 'events' significant to a user/group can be defined, similar content can be recommended to the users/groups, or customized video summaries generated on-the-fly.

The user tagging trends shown in Fig. 5 also illustrate that different social groups can react very differently to media content: vid 6 elicits a peaked negative response from technical users, whilst nontechnical users are primarily positively responsive. In contrast, vid 8 almost exhibits opposite responses from the two groups, particularly between 45-75 seconds. Such polar tagging trends between social groups affirm the importance of not only collecting and utilizing individual user media preferences for QoE but also collective responses within social contexts. Finally, while the measure of (1) equally weights the tags from each user and at each time, other weightings are also possible. For example, user's who regularly contribute meaningful tags ('lead users') within their social group could have their tags weighted higher, thus increasing the reliability of the opinion for the social group. Further, adaptive window lengths or shapes could also be applied, e.g. based on the detected location of scene boundaries or other content metadata.

\section{CONCLUSION}

This paper has described mechanisms that can be used to gather semantics that relate to a user's preferences when interacting with media content in social networks for use in adaptive multimedia systems. A system based on these mechanisms was implemented as a Facebook application that was used to run a series of subjective experiments. The results and discussion in this paper have highlighted that different social groups respond differently to media content, where users and groups further exhibit variable tagging behavior temporally within the same video. Immediate future work involves expanding Tag!t to collect more user media metadata to build semantics for use in a QoE adaptive multimedia system. Future user trials will include more users and employ a wider variety of video test content to subjectively evaluate a series of multimedia adaptation applications. Finally, we propose that the average user opinion, combined with metadata derived from automatic, signal processing-based analysis of the media content could be an effective measure of user QoP for input to a QoE adaptation system.

\section{ACKNOWLEDGEMENTS}

This work is funded by the Smart Services CRC.

\section{REFERENCES}

[1] "Youtube," 2008, Available at: http://www.youtube.com.

[2] "Facebook," 2008, Available at: http://www.facebook.com.

[3] "Myspace," 2008, Available at: http://www.myspace.com.

[4] Universal McCann, "Power to the People - Social Media Tracker Wave 4," 2009, Available at: http://universalmccann.bitecp.com/wave4/Wave4.pdf.

[5] S.R. Gulliver and G. Ghinea, "Defining user perception of distributed multimedia quality," ACM Trans. Multimedia Comput. Commun. Appl., vol. 2, 2006, pp. 241-257.

[6] J. Shin, D. Lee, and C.J. Kuo, Quality of service for internet multimedia, Prentice Hall Press, 2003.

[7] A. Vogel, B. Kerherve, G. von Bochmann, and J. Gecsei, "Distributed multimedia and QOS: a survey," IEEE Multimedia, vol. 2, 1995, pp. 10-19.

[8] M. van Der Schaar and Sai, "Cross-layer wireless multimedia transmission: challenges, principles, and new paradigms," Wireless Communications, IEEE [see also IEEE Personal Communications], vol. 12, 2005, pp. 58, 50.

[9] J. Thomas-Kerr, C. Ritz, and I. Burnett, "Semantic-Aware Delivery of Multimedia," ISCIT 2009, 2009, pp. 1498 1503.

[10] D. Jannach, K. Leopold, C. Timmerer, and H. Hellwagner, "A knowledge-based framework for multimedia adaptation," Applied Intelligence, vol. 24, Apr. 2006, pp. 109125.

[11] M. Prangl, T. Szkaliczki, and H. Hellwagner, "A Framework for Utility-Based Multimedia Adaptation," Circuits and Systems for Video Technology, IEEE Transactions on, vol. 17, 2007, pp. 719-728.

[12] R. Jain, "Quality of experience," Multimedia, IEEE, vol. 11, 2004, pp. 96-95.

[13] S. Davis, I. Burnett, and C. Ritz, "Using Social Networking and Collections to Enable Video Semantics Acquisition," to appear in IEEE Multimedia, 2009.

[14] "Flickr," 2008, Available at: http://www.flickr.com.

[15] Q. Li and S.C. Lu, "Collaborative Tagging Applications and Approaches," Multimedia, IEEE, vol. 15, 2008, pp. 14-21.

[16] S. Pfeiffer, C. Parker, and C. Schremmer, "Annodex: a simple architecture to enable hyperlinking, search $\backslash \&$ retrieval of time--continuous data on the Web," Proceedings of the 5th ACM SIGMM international workshop on Multimedia information retrieval, Berkeley, California: ACM, 2003, pp. 87-93. 